\title{
IDENTIFICATION AND CHARACTERISATION OF A CDNA CLONE FOR HISTONE H3 IN BARLEY
}

by

JAN CHOJECKI

Department of Physiology, Carlsberg Laboratory, Gamle Carlsberg Vej 10, DK-2500 Copenhagen Valby

Keywords: DNA sequence, polyadenylation, codon usage, 3' secondary structure, gene family

The 522 bp-long nucleotide sequence of a cDNA clone encoding the carboxyterminal part of a histone $\mathrm{H} 3$ in barley is presented. The cDNA represents a typical histone gene in that the coding sequence is highly conserved and it is reiterated, with 10-20 copies in the genome. However, unlike most histone mRNAs, the barley clone has an apparent poly(A) tail. There is degenerate homology with 3' consensus sequences of histone genes from other organisms, and a putative secondary loop structure which may represent a regulatory sequence. The codon usage in the amino acid coding region of the cDNA is highly skewed, with exclusively $\mathrm{C}$ or $\mathrm{G}$ being used in the third base position.

\section{INTRODUCTION}

The organisation and expression of histone genes have been extensively studied in animals, notably sea urchins, but only sparsely in plants (for reviews, see refs. 13, 15). The DNA and amino acid sequences of histone genes are very highly conserved among organisms, reflecting the importance of the histones as fundamental components of chromosome structure. Even in the non-coding flanking regions, there is considerable homology among histone genes. The genes are arranged in clusters containing containing one copy of each of the five different histone genes, H1, H2A, H2B, H3, and H4. These clusters are reiterated up to several hundred-fold in the genome, depending on the species. In most cases, histone mRNAs appear to lack the poly(A) tail characteristic of the greater proportion of eukaryotic messengers, although exceptions exist.
In plants the only histone DNA clones that have been identified are those for $\mathrm{H} 3$ and $\mathrm{H} 4$ histones in wheat $(22,23)$ and maize $(5)$ and $\mathrm{H} 2 \mathrm{~A}, \mathrm{H} 2 \mathrm{~B}$, and $\mathrm{H} 4$ in rice (24). All these clones were isolated from genomic DNA libraries. This paper reports the identification and initial characterisation of a cDNA clone for histone $\mathrm{H} 3$ from barley.

\section{MATERIALS AND METHODS}

The cDNA clone was identified in a cDNA library constructed from mRNA from scutella of Nudinka barley seeds at two days after the onset of germination. The details of the methods used for DNA sequence analysis, handling of sequence data, and DNA hybridisation are described in the accompanying paper (6). The protein sequence database screened by computer analysis was the Protein Identification

Abbreviations: $b p=$ base pair; $\mathbf{k b p}=$ kilobase pairs 
1

AAG AGC ACC GAG CTG CTG ATC CGC AAG CTC CCG TTC CAG CGC CTG LYS SER THR GLU LEU LEU ILE ARG LYS LEU PRO PHE GLN ARG LEU 56

50

GTG AGG GAG ATC GCG CAG GAC TTC AAG ACC GAC CTC AGG TTC CAG VAL ARG GLU ILE ALA GLN ASP PHE LYS THR ASP LEU ARG PHE GLN

100

TCC CAC GCC GTG CTG GCC CTC CAG GAG GCC GCC GAG GCG TAC CTC SER HIS ALA VAL LEU ALA LEU GLN GLU ALA ALA GLU ALA TYR LEU

150

100

GTC GGG CTG TTC GAG GAC ACC AAC CTG TGC GCC ATC CAC GCC AAG VAL GLY LEU PHE GLU ASP THR ASN LEU CYS ALA ILE HIS ALA LYS

$$
200
$$

CGC GTC ACC ATC ATG CCC AAG GAC ATC CAG CTC GCC CGC CGC ATC ARG VAL THR ILE MET PRO LYS ASP ILE GLN LEU ALA ARG ARG ILE 250

CGC GGG GAG CGC GCC TAA GCCACCCAGAGCGCTGCATTCGGGAGCGATGACAC ARG GLY GLU ARG ALA

300

CGTTCGCCAGCATTAGTGTAGTTGATTGGCTTTCCTTGTCCAGATATGCGTCTTGTGGT 350

TCGTTGTAGAAACCCTGGTTGGTTGGTTCCCGTAGTTACAGAGACTTTTCTGCTTAAGT 400 450 GGTTTTGGTTTGCGGTGTTGCAAACCGATGCTTACTGTGATGCAAATTGTTGGTTAATG 500 TAGTGTTGATTGACAATTATCGATGGATGAACTTGTGGTGTTGCGTAGTTAAAAAAAAA

\section{AAAAAAAA}

Figure 1. Nucleotide sequence of cDNA clone encoding barley H3 histone, and predicted amino acid sequence. The amino acid sequence is numbered according to the system of DE LANGE et al. (7).

Resource (NBRF, Georgetown University Medical Centre, 3900 Reservoir Road, N.W., Washington DC, 20007).

\section{RESULTS}

The identification of the cDNA clone for barley histone $\mathrm{H} 3$ was made on the basis of the 


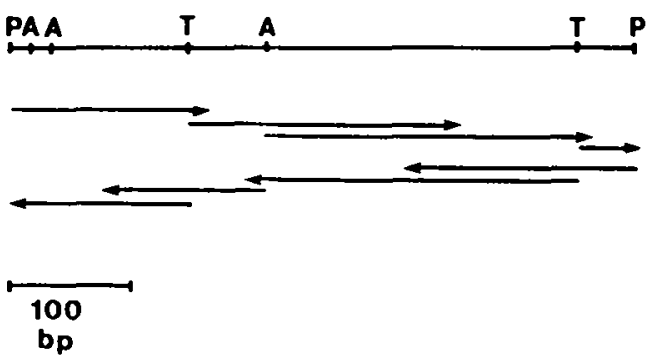

Figure 2. Strategy used for sequencing barley $\mathrm{H} 3$ histone cDNA.

P = Pst I, A = Alu I, T = Taq I restriction sites.

almost complete homology between the amino acid sequence predicted from its DNA sequence, and the $\mathrm{H} 3$ histone sequences from other organisms, represented on the PIR database being searched.

The cDNA (Figure 1) is 522 bp long, containing DNA coding for 80 amino acids of the carboxyterminal part of the $\mathrm{H} 3$ protein, a termination codon and 279 nucleotides of 3 ' non-coding region. The nucleotide sequence was determined by the strategy described in Figure 2 . The polypeptide encoded by the wheat $\mathrm{H} 3$ gene is 135 amino acids long (23). Thus the barley $\mathrm{H} 3$ cDNA described here lacks the DNA coding for the 55 amino-terminal amino acids, plus any $5^{\prime}$ non-translated sequence.

The 3' non-coding region contains a block of 17 " A"s at the 3' end. This may represent a true poly(A) tail, but the possibility that it is an

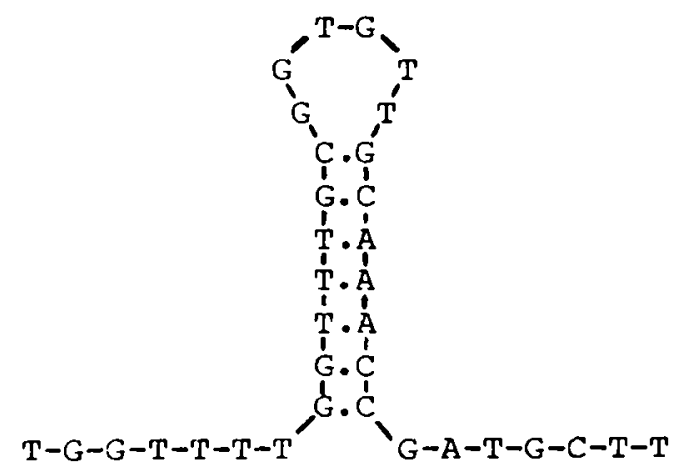

Figure 3. Putative secondary structure in 3 ' non-coding region of barlcy $\mathrm{H} 3$ histone mRNA.

$\Delta \mathrm{G}, 25^{\circ} \mathrm{C}=-12.8 \mathrm{kcal} / \mathrm{mol}$.

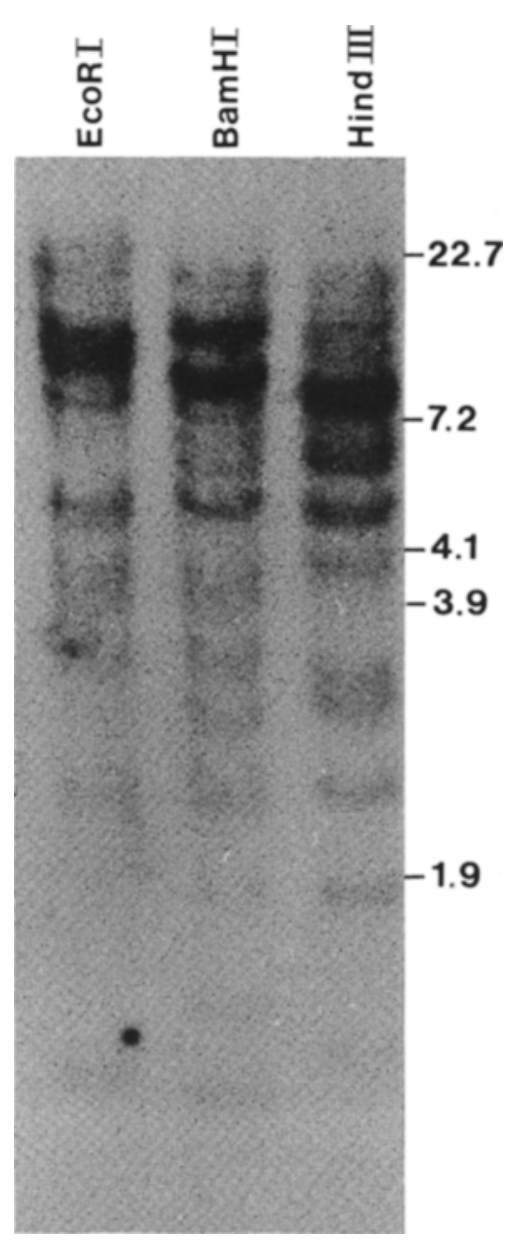

Figure 4. Southern hybridisation analysis.

Barley genomic DNA (cv. Carlsberg II), digested with different restriction endonucleases, and probed with the nick-translated $\mathrm{H} 3$ histone cDNA insert. Fragment sizes were estimated using the Hind III restriction fragments of $\lambda$-Wes/ $\lambda \mathrm{b}$ as markers, indicated in kbp.

artefact, resulting from oligo-d(T) priming on an A-rich region during first strand cDNA synthesis, cannot be excluded. There is also an inverted repeat that could give rise to a secondary structure (Figure 3 ). The structure has a theoretical thermodynamic stability of -12.8 $\mathrm{kcal} / \mathrm{mol}$, as calculated using the method of Tinoco et al. (25).

Hybridisation of the $\mathrm{H} 3$ cDNA to digested barley genomic DNA (Figure 4) shows a number of hybridising fragments of different size and 
Second letter

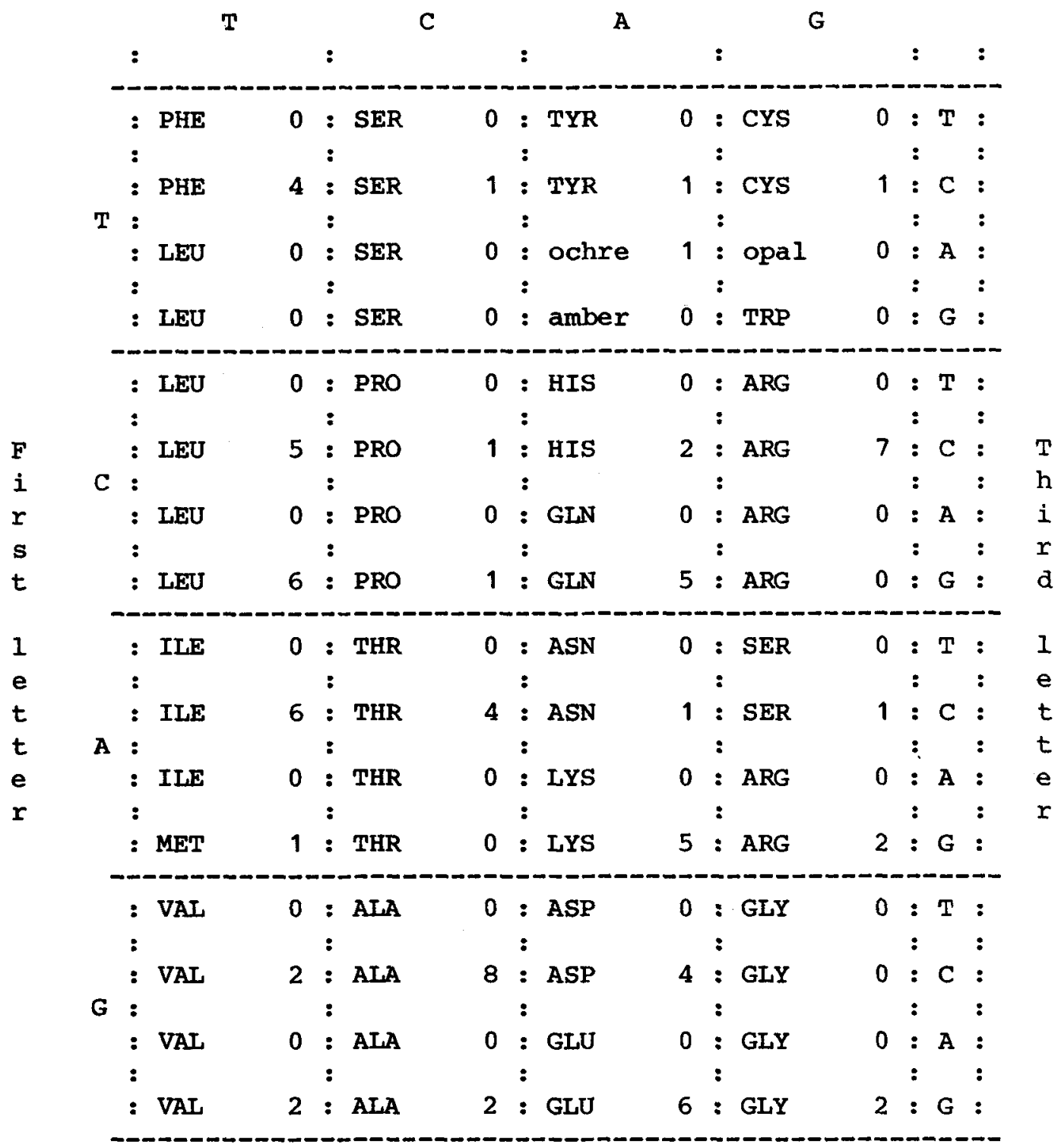

Figure 5. Codon usage of barley $\mathrm{H} 3$ histone cDNA clone.

intensity. Based on the assumption that the most weakly hybridising fragments represent single copies of an $\mathrm{H} 3$ gene, the pattern suggests a reiteration frequency of 10-20 copies per haploid barley genome. This is comparable to the copy number of $\mathrm{H} 3$ in wheat of $80-100$ copies per hexaploid genome (23) and in maize of 30-40 copies per haploid genome (5).

\section{DISCUSSION}

The nucleotide sequence of the barley $\mathrm{H} 3$ cDNA is highly homologous in the coding region with those of wheat $\mathrm{H} 3(90 \%$, ref. 23$)$ and maize $(88 \%$, ref. 5$)$. Most of the nucleotide differences are in the third base position of the codons, with the effect that only two amino acids differ between barley and the H3s of wheat and 
Figure 6. Homologies between 3' non-coding region of histone genes from different species.

(1) Sequence data from sea urchin, Psammechinus miliaris, H3 histone gene (4) showing consensus sequence shared by sea urchin histone genes underlined) and putative dyad symmetry (arrows).

(2), (3), and (4) 3' non-coding regions of barley H3, wheat $\mathrm{H3}$ (23) and wheat $\mathrm{H} 4$ (22) histone genes showing regions of homology with sea urchin consensus, and putative dyad symmetries.

maize. These are histidine instead of serine (residue 87) and leucine instead of serine (wheat) or alanine (maize) at residue 90 . The wheat and pea (17) amino acid sequences are identical, so it is interesting that wheat and barley $\mathrm{H} 3$ differ at all. There are only four amino acid differences throughout the entire $\mathrm{H} 3$ sequences of wheat (and pea) and calf (7), of which one is the residue at position 90 (methionine in calf) which is also different in both barley and maize. Position 90 is clearly not under such strong conservation pressure as other parts of the sequence. However, since two forms of histone $\mathrm{H} 3$ which differ in residue 96 have been reported for pea (17) and calf (16), it is probable that two or more different histone $\mathrm{H} 3$ genes are operational in any individual, producing the microheterogeneities observed at the protein level.

The codon usage (Figure 5) is highly biased with respect to the third, wobble, base of each codon. Throughout the 80 codons represented in the cDNA insert, the wobble base is exclusively $\mathbf{G}$ or $\mathrm{C}$. Similarly, the $\mathrm{H} 3$ genes of wheat, maize and chicken $(5,10,23)$ have $G$ or $C$ in the third base in most codons, but not all codons. Sequence data from several different sea urchin histone genes $(20,21)$ show a preference for $\mathrm{C}$ and, to a lesser extent $G$, at the third base position, but not to the same extent as that seen in barley, wheat, maize and chicken. Codon usage peculiarities are not organism-specific (eg. compare codon usage of barley $\mathrm{H} 3$, here, with that of GAPDH in the accompanying paper). Furthermore, although "tissue-specific" patterns of codon usage in different mRNAs have been noted (12), messengers in the same cell may have different patterns of preference (e.g. mRNAs for rabbit $\alpha$-globin versus $\beta$-globin $(9$, 14) or, in chicken heart muscle, GAPDH versus myosin mRNAs (8)). Despite these observations, it is unclear as yet whether biased codon usages are of significance for the translation rate of specific mRNAs, either because of effects on mRNA stability or because of differential tRNA availability.

Histone mRNAs are the most prominent species of non-polyadenylated messengers (1). However, poly (A)+ histone mRNAs have been reported in Xenopus oocytes (19) and yeast (11), as well as the barley mRNA described here. In addition, a small fraction of the histone $\mathrm{mRNA}$ of HeLa cells, sea urchins and clams may be polyadenylated (for review, see ref. 15). In Xenopus, after fertilization of oocytes, the histone mRNA is predominantly non-polyadenylated (19). Thus, the detection of polyadenylated histone messages may depend critically upon the developmental stage investigated. It will be possible to study this phenomenon further, using the barley $\mathrm{H} 3 \mathrm{cDNA}$ as a probe.

The absence of the putative polyadenylation signal AATAAA (18) is a feature of all histone genes whose 3 ' regions have been sequenced, with the possible exception of yeast H4 (13). 
Since poly(A)+ histone mRNAs exist without this polyadenylation signal, it is possible that AATAAA may not be a universal signal $(1,3)$.

Apart from the AATAAA consensus, most eukaryotic $m R N A s$ have very little or no homology among their ' 3' non-translated sequences. In contrast, however, the 3 ' regions of histone mRNAs (or 3' flanking regions of genes) lack the AATAAA consensus but do have blocks of considerable homology among different histones in different species of sea urchin, Xenopus, Drosophila, chicken and mouse (13). One of these blocks can form a putative secondary structure (4), which acts as a recognition signal involved in the termination of transcription and processing of the 3' end of the transcript (2). There is some homology between this sequence and the barley $\mathrm{H} 33$ ' non-coding region (Figure 6) but the dyad structure cannot be formed by the barley sequence. However, a different sequence, lying 3' to the region of homology with the sea urchin consensus, can form a dyad structure (Figure 3 ) with a stability similar to that predicted for the putative loops in sea urchin histone 3' regions (4). Wheat $\mathrm{H} 3$ and $\mathrm{H} 4$ genes $(22,23)$ both have degenerate homology in the 3' flanking region with the sea urchin consensus and both have inverted repeats which could form secondary structures (albeit of low theoretical stability) downstream of this region (Figure 6). It is also intriguing that these putative secondary structures in wheat and barley histone 3' sequences all contain a common tetranucleotide, TGTT, in the unpaired part of the loop.

While histone protein synthesis is closely related to DNA replication, the relationship between histone transcript synthesis/turnover and DNA replication may be more complicated (for review, see ref. 15). The cDNA for barley histone $\mathrm{H} 3$ can be used as a probe to study the regulation of expression of the $\mathrm{H} 3$ gene(s) and it may be useful as a marker for tissue engaged in mitotic activity. In this respect, the isolation of the clone in a cDNA library made from scutellar mRNA during germination, may reflect some contamination by adjacent (mitotic) embryo tissue or the occurrence of endomitotic chromosome duplication on this tissue, highly active in enzyme secretion and transport of sugars and amino acids.

\section{ACKNOWLEDGEMENTS}

The author is grateful to ANDERs BRANDT and GEOFF FINCHER for providing the cDNA library, and to ANDERS BRANDT and Prof. DITER VON WETTSTEIN for critical reading of the manuscript. The work was supported by the Biomolecular Engineering Programme of the Commission of European Communities with Training Contract No. GBI-0172-DK and Research Contract No. GBI-4-024-DK to Prof. D. VON WETTSTEIN.

\section{REFERENCES}

1. Baralle, F. E.: The functional significance of leader and trailer sequences in eukaryotic mRNAs. Int. Rev. Cyt. 81, 71-106 (1983)

2. Birchmeier, C., R. Grosschedl \& M. L. BirnsTIEL: Generation of authentic 3' termini of an $\mathrm{H} 2 \mathrm{~A}$ mRNA in vivo is dependent on a short inverted DNA repeat and on spacer sequences. Cell 28 , 739-745 (1982)

3. Birnstiel, M. L., M. Busslinger \& K. Strub: Transcription termination and 3' processing. Cell, 41, 349-359 (1985)

4. Busslinger, M., R. Portmann \& M. L. BirnsTIEL: A regulatory sequence near the 3' end of sea urchin histone genes. Nucleic Acids Res. 6, 2997 3008 (1979)

5. Chaubet, N, G. Philipps, M. Chaboute, M. EhlING \& C. GIGOT: Genomic organisation of corn histone $\mathrm{H} 3$ and $\mathrm{H} 4$ genes. Plant Mol. Biol. 6, 253-263 (1986)

6. CHOJECKI, J.: Identification and characterisation of a cDNA clone for cytosolic glyceraldehyde-3phosphate dehydrogenase in barley. Carlsberg Res. Commun. 51, 203-210 (1986)

7. Delange, R. J., J. A. HoOper \& E. L. Smith: Complete amino acid sequence of calf-thymus histone III. Proc. Nat. Acad. Sci. USA 69, 882-884 (1972)

8. Domdey, H., K. Wiebauer, H. Klapthor \& H. ARNOLD: Sequence analysis of the cloned mRNA coding for glyceraldehyde-3-phosphate dehydrogenase from chicken heart muscle. Eur. J. Biochem. 131, 129-135(1983)

9. Efstratiadis, A., F. C. Kafatos \& T. Maniatis: The primary structure of structure rabbit $\beta$-globin mRNA as determined from cloned DNA. Cell 10, 571-585 (1977)

10. Engel, J. D., B. J. Sugarman \& J. B Dodgson: A chicken histone gene contains intervening sequences. Nature 297, 434-436 (1982)

11. FAHRNER, K., J. YARGER \& L. HeReford: Yeast 
histone mRNA is polyadenylated. Nucleic Acids Res. 8, 5725-5738 (1980)

12. Fincher, G. B., P. A. Lock, M. M. Morgan, K. Lingelbach, R. Wettenhall, J. Mercer, A. BRANDT \& K. K. THOMSEN: Primary structure of the $(1 \rightarrow 3,1 \rightarrow 4)-\beta$-D-glucan 4-glucanohydrolase from barley aleurone. Proc. Nat. Acad. Sci. USA 83, 2081-2085 (1986)

13. HentsChel, C. C.\& M. L. Birnstiel: The organisation and expression of histone gene families. Cell 25, 301-313 (1981)

14. Heindell, H. C., A. Liu, G. V. Paddock, G. M. STUdNicka \& W. A. SAlser: The primary sequence of rabbit $\alpha$-globin mRNA. Cell 15, 43-54 (1978)

15. KeDES, L. H.: Histone genes and histone messengers. Ann. Rev. Biochem. 48, 837-870 (1979)

16. Marzluff, W. F., L. A. SAunders, D. M. Miller \& K. S. MCCARTY: Two chemically distinct forms of calf-thymus histone H3. J. Biol. Chem. 247, 2026-2033 (1972)

17. Patthy, L. \& E. L. Smith: Histone III. V. The amino acid sequence of pea embryo histone III. J. Biol. Chem. 248, 6834-6840 (1973)

18. Proudfoot, N. J.\& G.G. BrownleE: 3' Non-coding region sequences in eukaryotic messenger RNA. Nature 263, $211-214$ (1976)

19. RudermanN, J. V. \& M. L. Pardue: A portion of all major classes of histone mRNA in amphibian oocytes is polyadenylated. J. Biol. Chem. 253, 2018-2025 (1978)

20. SChaffNer, W., G. Kunz, H. Daetwyler, J. TelFORD, H. O. SMITH \& M. L. BIRNSTIEL: Genes and spacers of cloned sea urchin histone DNA analysed by sequencing. Cell 14, 655-671 (1978)

21. Sures, I., A. MaXam, R. H. Cohn \& L. H. Kedes: Identification and location of the histone $\mathrm{H} 2 \mathrm{~A}$ and $\mathrm{H} 3$ genes by sequence analysis of sea urchin ( $\mathrm{S}$. purpuratus) DNA cloned in E. coli. Cell 9, 495-502 (1976)

22. Tabata, T., K. Sasaki \& M. Iwabuchi: The structural organisation and DNA sequence of a wheat histone H4 gene. Nucleic Acids Res. 11, 5865-5875 (1983)

23. Tabata, T., M. Fukasawa \& M. Iwabuchi: Nucleotide sequence and genomic organisation of a wheat histone H3 gene. Mol. Gen. Genet. 196, 397-400 (1984)

24. Thomas, G. \& J. D. Padayatty: Restriction map and partial sequence of a rice DNA fragment carrying histone genes $\mathrm{H} 2 \mathrm{~A}, \mathrm{H} 2 \mathrm{~B}$ and $\mathrm{H} 4$. Ind. J. Biochem. 21, 1-6 (1984)

25. Tinoco, I., P. Borer, B. Dengler, M. Levine, $\mathbf{O}$. Uhlenbeck, D. Crothers \& J. Gralla: Improved estimation of secondary structure in ribonucleic acids. Nature New Biology 246, 40-41 (1973) 